\title{
Bias Correction and Modified Profile Likelihood under the Wishart Complex Distribution
}

\author{
Abraão D. C. Nascimento, Alejandro C. Frery, Senior Member, and Renato J. Cintra, Senior Member
}

\begin{abstract}
This paper proposes improved methods for the maximum likelihood (ML) estimation of the equivalent number of looks $L$. This parameter has a meaningful interpretation in the context of polarimetric synthetic aperture radar (PolSAR) images. Due to the presence of coherent illumination in their processing, PolSAR systems generate images which present a granular noise called speckle. As a potential solution for reducing such interference, the parameter $L$ controls the signal-noise ratio. Thus, the proposal of efficient estimation methodologies for $L$ has been sought. To that end, we consider firstly that a PolSAR image is well described by the scaled complex Wishart distribution. In recent years, Anfinsen et al. derived and analyzed estimation methods based on the ML and on trace statistical moments for obtaining the parameter $L$ of the unscaled version of such probability law. This paper generalizes that approach. We present the second-order bias expression proposed by Cox and Snell for the ML estimator of this parameter. Moreover, the formula of the profile likelihood modified by Barndorff-Nielsen in terms of $L$ is discussed. Such derivations yield two new ML estimators for the parameter $L$, which are compared to the estimators proposed by Anfinsen et al. . The performance of these estimators is assessed by means of Monte Carlo experiments, adopting three statistical measures as comparison criterion: the mean square error, the bias, and the coefficient of variation. Equivalently to the simulation study, an application to actual PolSAR data concludes that the proposed estimators outperform all the others in homogeneous scenarios.
\end{abstract}

\section{INTRODUCTION}

Multilook polarimetric synthetic aperture radar (PolSAR) is a technology which has proven to be an effective tool for geophysical remote sensing [1]. This particular system operates by sending electromagnetic pulses of several polarizations towards a remote target; then the returning echoes are captured and registered. This process is characterized by the use of coherent illumination, which affects the obtained images with a granular noise called 'speckle'. Therefore, a specialized modeling is required for processing and analyzing such images.

In this context, the scaled complex Wishart distribution has been suggested as a successful approach for modeling the backscattered PolSAR signal. This distribution is equipped

This work was supported by CNPq, Fapeal and FACEPE, Brazil.

A. D. C. Nascimento is with the Departamento de Estatística, Universidade Federal de Pernambuco, Cidade Universitária, 50740-540, Recife, PE, Brazil, e-mail: abraao.susej@gmail.com

This work was partially funded by Conicet, CNPq, Fapeal and Capes

A. C. Frery is with the LaCCAN - Laboratório de Computação Científica e Análise Numérica, Universidade Federal de Alagoas, BR 104 Norte km 97, 57072-970, Maceió, AL, Brazil, e-mail: acfrery@gmail.com

R. J. Cintra is with the Signal Processing Group, Departamento de Estatística, Universidade Federal de Pernambuco, Cidade Universitária, 50740540, Recife, PE, Brazil, e-mail: rjdsc@ieee.org with two parameters: a covariance matrix and the number of looks $(L)$. The former quantity is directly related to the data structure and is defined over the set of positive-definite Hermitian matrices [2]. On the other hand, parameter $L$ is defined as the mean number of statistically independent samples represented by each pixel. Indeed, parameter $L$ is related to the signal-to-noise ratio and the speckle noise is less severe for large values of $L$ [3], [4].

Several studies have addressed the issue of estimating $L$ [5], [6]. Despite the various existing approaches, the maximum likelihood (ML) method is often selected as the method of choice. This is mainly due to its good asymptotic properties, such as consistency and convergence in distribution to the Gaussian law [7].

However, the ML estimator in finite samples is usually biased with respect to the true parameter value. Indeed, the ML estimator bias has order $O\left(N^{-1}\right)$, where $N$ is the sample size and $O(\cdot)$ is Landau notation to represent order. In practice, this quantity is not commonly considered because its value is negligible when compared to the standard error, which has order $O\left(N^{-1 / 2}\right)$. However, such biases can be substantial in situations where small or moderate sample sizes are used. Thus, analytic expressions for the ML estimator bias have been sought in order to derive a more accurate, corrected estimator for finite sample sizes [8], [9].

In [10], Cox and Snell proposed a general formula for the second-order bias of the ML estimator. Several works have considered this formula as a mean to obtain improved estimators for parameters of several distributions [9]. Vasconcellos et al. [8] applied it to the univariate $\mathcal{G}^{0}$ distribution, which is often used for modeling synthetic aperture radar (SAR) images with one polarization channel [11]. Pianto and Cribari-Neto [12] proposed a bias correction approach for the roughness parameter of the $\mathcal{G}^{0}$ distribution.

More accurate ML estimators can also be derived by means of the modified profile likelihood [13, chapter 9]. In this approach, an improved parameter estimation can be achieved by maximizing an adjusted profiled likelihood function [14]. Barndorff-Nielsen proposed a successful adjustment for the profile likelihood in [15]. This methodology could offer parametric inference techniques suitable for several distributions. The Birnbaum-Saunders distribution [16] and elliptical structural models [17] are illustrative examples. This approach has also been employed in SAR data models. Silva et al. [18] proposed point estimation and hypothesis test methods for the roughness parameter of the $\mathcal{G}^{0}$ distribution based on modified profile likelihood.

Anfinsen et al. [5] proposed three methods for estimating 
the number of looks. Their methods were favorably compared with classic methods based on fractional moment and the coefficient of variation. Assuming that PolSAR images are well modeled by the complex Wishart distribution, the ML estimator was found to excel when compared to other classic techniques. Additionally, such estimators were submitted to bias analysis by means of jackknife and bootstrap techniques [5]. However, these techniques are computationally intensive and are prone to inaccuracies when small sample sizes are considered [19].

The aim of this paper is three-fold. First, we derive a second-order bias expression for the ML estimator of parameter $L$ and propose corrected estimator for $L$ following CoxSnell approach [10]. Second, considering the scaled complex Wishart law, we propose a new estimator for $L$ according Barndorff-Nielsen correction [15]. Third, the performance of the proposed corrected ML estimators is quantified and compared to the estimators listed in literature. This assessment is performed according to Monte Carlo experiments at several different simulation scenarios. Actual PolSAR data are also considered and analyzed.

The remainder of this paper is structured as follows. In Section II, we review the scaled complex Wishart distribution, which is the probability model considered in this work. Section III discusses Cox-Snell and Barndorff-Nielsen methodologies for obtaining corrected ML estimators. In Section IV, the new corrected estimators are introduced and mathematically described. Subsequently, the derived estimators are analyzed and compared with existing literature techniques in Section IV. Finally, conclusions are summarized in Section VI.

\section{SCALED COMPLEX WishaRT Distribution AND ML ESTIMATION}

\section{A. Scaled Complex Wishart distribution}

The polarimetric coherent information associates to each image pixel a $2 \times 2$ complex matrix. The entries of such matrix are $S_{\mathrm{VV}}, S_{\mathrm{VH}}, S_{\mathrm{HV}}$, and $S_{\mathrm{HH}}$, where $S_{i j}$ represents the backscattered signal for the $i$ th transmission and $j$ th reception linear polarization, for $i, j=\mathrm{H}, \mathrm{V}$. Under the reciprocity theorem conditions [20], the scattering matrix is often simplified into a three-component vector, because $S_{\mathrm{HV}}=S_{\mathrm{VH}}$. Thus, we have the following scattering vector

$$
\left[\begin{array}{lll}
S_{\mathrm{VV}} & \sqrt{2} S_{\mathrm{VH}} & S_{\mathrm{HH}}
\end{array}\right]^{\top},
$$

where the superscript ${ }^{\top}$ denotes vector transposition. This three-component representation is detailed in [21].

In general, we may consider a system with $m$ polarizations. Thus the associated complex random vector is denoted by:

$$
\boldsymbol{s}=\left[\begin{array}{llll}
S_{1} & S_{2} & \cdots & S_{m}
\end{array}\right]^{\top},
$$

where $S_{j}, j=1,2, \ldots, m$, represents the random variables associated with each polarization channel. This particular random vector has been successfully modeled by the multivariate complex Gaussian distribution with zero-mean, as discussed in [22]. Thus, the single-look PolSAR data representation is essentially given by $s$.
Multilook polarimetric speckled data obtained from scattered signal is commonly defined by the estimated sample covariance matrix [5], which is given by

$$
\boldsymbol{Z}=\frac{1}{L} \sum_{i=1}^{L} \boldsymbol{s}_{i} \boldsymbol{s}_{i}^{\mathrm{H}}
$$

where the superscript $\mathrm{H}$ represents the complex conjugate transpose of a vector, $\boldsymbol{s}_{i}, i=1,2, \ldots, L$, are scattering vectors, and $L$ is the number of looks. Matrix $Z$ is Hermitian positive definite and follows a scaled complex Wishart distribution [5]. Having $\boldsymbol{\Sigma}$ and $L$ as parameters, the scaled complex Wishart distribution is associated to the following probability density function [2]:

$$
f_{\boldsymbol{Z}}\left(\boldsymbol{Z}^{\prime} ; \boldsymbol{\Sigma}, L\right)=\frac{L^{m L}\left|\boldsymbol{Z}^{\prime}\right|^{L-m}}{|\boldsymbol{\Sigma}|^{L} \Gamma_{m}(L)} \exp \left(-L \operatorname{tr}\left(\boldsymbol{\Sigma}^{-1} \boldsymbol{Z}^{\prime}\right)\right),
$$

where $\Gamma_{m}(L)=\pi^{m(m-1) / 2} \prod_{i=0}^{m-1} \Gamma(L-i)$ for $L \geq m, \Gamma(\cdot)$ is the gamma function, $\operatorname{tr}(\cdot)$ represents the trace operator, $|\cdot|$ denotes the determinant operator, $\boldsymbol{\Sigma}$ is the covariance matrix associated to $s$,

$$
\begin{aligned}
\Sigma & =\mathrm{E}\left(\boldsymbol{s} s^{*}\right) \\
& =\left[\begin{array}{cccc}
\mathrm{E}\left(S_{1} S_{1}^{*}\right) & \mathrm{E}\left(S_{1} S_{2}^{*}\right) & \cdots & \mathrm{E}\left(S_{1} S_{m}^{*}\right) \\
\mathrm{E}\left(S_{2} S_{1}^{*}\right) & \mathrm{E}\left(S_{2} S_{2}^{*}\right) & \cdots & \mathrm{E}\left(S_{2} S_{m}^{*}\right) \\
\vdots & \vdots & \ddots & \vdots \\
\mathrm{E}\left(S_{m} S_{1}^{*}\right) & \mathrm{E}\left(S_{m} S_{2}^{*}\right) & \cdots & \mathrm{E}\left(S_{m} S_{m}^{*}\right)
\end{array}\right],
\end{aligned}
$$

and superscript ${ }^{*}$ means the conjugate of a complex number. The first moment of $\boldsymbol{Z}$ satisfies $\mathrm{E}(\boldsymbol{Z})=\boldsymbol{\Sigma}$ [5], where $\mathrm{E}(\cdot)$ is the statistical expectation operator. We denote $Z \sim \mathcal{W}(\Sigma, L)$ to indicate that $Z$ follows the scaled complex Wishart distribution.

\section{B. Estimation of Scaled Complex Wishart Distribution Param-} eters

Let $\left\{\boldsymbol{Z}_{1}, \boldsymbol{Z}_{2}, \ldots, \boldsymbol{Z}_{N}\right\}$ be a random sample from $\boldsymbol{Z} \sim$ $\mathcal{W}(\boldsymbol{\Sigma}, L)$. The ML estimators for $\boldsymbol{\Sigma}$ and $L$, namely $\widehat{\boldsymbol{\Sigma}}_{\mathrm{ML}}$ and $\widehat{L}_{\mathrm{ML}}$, respectively, are quantities that maximize the loglikelihood function associated to the Wishart distribution, which is given by [5], [23]:

$$
\begin{aligned}
\ell(\boldsymbol{\theta})= & m N L \log L+(L-m) \sum_{k=1}^{N} \log \left|\boldsymbol{Z}_{k}\right|-L N \log |\boldsymbol{\Sigma}| \\
& -N \frac{m(m-1)}{2} \log \pi-N \sum_{k=0}^{m-1} \log \Gamma(L-k) \\
& -N L \operatorname{tr}\left(\boldsymbol{\Sigma}^{-1} \overline{\boldsymbol{Z}}\right),
\end{aligned}
$$

where $\bar{Z}=N^{-1} \sum_{k=1}^{N} \boldsymbol{Z}_{k}, \boldsymbol{\theta}=\left[\begin{array}{ll}\boldsymbol{\sigma}^{\top} & L\end{array}\right]^{\top}, \boldsymbol{\sigma}=\operatorname{vec}(\boldsymbol{\Sigma})$, and $\operatorname{vec}(\cdot)$ is the column stacking vectorization operator.

In a previous work [23], we have showed that $\widehat{\Sigma}_{\mathrm{ML}}$ is given by the sample mean,

$$
\widehat{\mathbf{\Sigma}}_{\mathrm{ML}}=\overline{\boldsymbol{Z}}
$$


and $\widehat{L}_{\mathrm{ML}}$ satisfies the following non-linear equation:

$$
m \log \widehat{L}_{\mathrm{ML}}+\frac{1}{N} \sum_{k=1}^{N} \log \left|\boldsymbol{Z}_{k}\right|-\log |\overline{\boldsymbol{Z}}|-\psi_{m}^{(0)}\left(\widehat{L}_{\mathrm{ML}}\right)=0,
$$

where $\psi_{m}^{(0)}(\cdot)$ is the zeroth order term of the $v$ th-order multivariate polygamma function given by

$$
\psi_{m}^{(v)}(L)=\sum_{i=0}^{m-1} \psi^{(v)}(L-i),
$$

$\psi^{(v)}(\cdot)$ is the ordinary polygamma function expressed by

$$
\psi^{(v)}(L)=\frac{\partial^{v+1} \log \Gamma(L)}{\partial L^{v+1}},
$$

for $v \geq 0$. Function $\psi^{(0)}(\cdot)$ is known as the digamma function.

In above expression (2) a closed-form analytical solution for $\widehat{L}_{\mathrm{ML}}$ [5], [23] could not be identified. Nevertheless, $\widehat{L}_{\mathrm{ML}}$ can be obtained via the Newton-Raphson numerical optimization method [24]. A detailed account on the performance of $\widehat{L}_{\mathrm{ML}}$ is provided in [5]. However, estimator $L_{\mathrm{ML}}$ is not adequate for applications based on small sample sizes, such as filtering methods for image restoration [25], [26]. This is due to the fact that the ML estimator is only guaranteed to be asymptotically unbiased; therefore, techniques which depend on ML estimates under small samples may suffer from pronounced bias.

A convenient estimator based on the method of moments for the equivalent number of looks (ENL) was proposed in [27] and suggested for both single-polarization and PolSAR data [5]. However, for that particular method, in the PolSAR case, the cross-terms of the estimated sample covariance matrix are not considered [5], which means that potentially useful information is simply being discarded.

To address this issue, Anfinsen et al. [5] proposed two estimators based on trace moment, whose expressions are given by:

$$
\widehat{L}_{\mathrm{MM} 1}=\frac{\operatorname{tr}(\overline{\boldsymbol{Z}} \overline{\boldsymbol{Z}})}{N^{-1} \sum_{i=1}^{N} \operatorname{tr}\left(\boldsymbol{Z}_{i}\right)^{2}-\operatorname{tr}(\overline{\boldsymbol{Z}})^{2}}
$$

and

$$
\widehat{L}_{\mathrm{MM} 2}=\frac{\operatorname{tr}(\overline{\boldsymbol{Z}})^{2}}{N^{-1} \sum_{i=1}^{N} \operatorname{tr}\left(\boldsymbol{Z}_{i} \boldsymbol{Z}_{i}\right)-\operatorname{tr}(\overline{\boldsymbol{Z}} \overline{\boldsymbol{Z}})} .
$$

These estimators are based on the derivations proposed by Maiwald and Kraus [28].

\section{Corrected ML estimation Methods}

Several works have addressed the ML estimator properties in finite sample for SAR data modeling [8], [12], [18]. ML estimation for other statistical models has also been proposed [9], [16], [17]. In this section, we describe two methodologies for obtaining improved ML estimators. The first one is based on the ML estimator second-order bias according to the Cox and Snell general formula [10]. The second method is based on the modified profile likelihood and was proposed by Barndorff-Nielsen [15].

\section{A. Mathematical Framework}

In this subsection, we present the mathematical framework required for both methods above referred to.

Let $\left\{\boldsymbol{Z}_{1}, \boldsymbol{Z}_{2}, \ldots, \boldsymbol{Z}_{N}\right\}$ be a random sample from a random positive-definite Hermitian matrix $Z$ equipped with a given density $f_{\boldsymbol{Z}}\left(\boldsymbol{Z}^{\prime} ; \boldsymbol{\theta}\right)$, where $\boldsymbol{Z}^{\prime}$ represents possible outcomes and $\boldsymbol{\theta}=\left[\begin{array}{llll}\theta_{1} & \theta_{2} & \cdots & \theta_{p}\end{array}\right]^{\top}$ is the parameter vector with length $p$.

The associated log-likelihood function based on above observations is given by

$$
\ell(\boldsymbol{\theta})=\sum_{k=1}^{N} \log f_{\boldsymbol{Z}}\left(\boldsymbol{Z}_{k} ; \boldsymbol{\theta}\right)
$$

The derivatives of the log-likelihood play an important role in ML estimation. For the sake of compactness, we adopt the following notation:

$$
\begin{aligned}
U_{\theta_{i}} & =\frac{\partial}{\partial \theta_{i}} \ell(\boldsymbol{\theta}), \\
U_{\theta_{i} \theta_{j}} & =\frac{\partial^{2}}{\partial \theta_{i} \partial \theta_{j}} \ell(\boldsymbol{\theta}), \\
U_{\theta_{i} \theta_{j} \theta_{k}} & =\frac{\partial^{3}}{\partial \theta_{i} \partial \theta_{j} \partial \theta_{k}} \ell(\boldsymbol{\theta}),
\end{aligned}
$$

for $i, j, k=1,2, \ldots, p$. Accordingly, we denote the cumulants for the log-likelihood derivatives as [9]:

$$
\begin{aligned}
\kappa_{\theta_{i} \theta_{j}} & =\mathrm{E}\left(U_{\theta_{i} \theta_{j}}\right), \quad \kappa_{\theta_{i}, \theta_{j}}=\mathrm{E}\left(U_{\theta_{i}} U_{\theta_{j}}\right), \\
\kappa_{\theta_{i} \theta_{j} \theta_{k}} & =\mathrm{E}\left(U_{\theta_{i} \theta_{j} \theta_{k}}\right), \quad \kappa_{\theta_{i}, \theta_{j} \theta_{k}}=\mathrm{E}\left(U_{\theta_{i}} U_{\theta_{j} \theta_{k}}\right) .
\end{aligned}
$$

As a direct consequence, the elements of the Fisher information matrix associated to $\boldsymbol{\theta}$ are $\kappa_{\theta_{i}, \theta_{j}}$. Additionally, we denote the elements of the inverse of the Fisher information matrix as $\kappa^{\theta_{i}, \theta_{j}}$.

Moreover, the first derivative of the cumulants with respect to the parameters is denoted by:

$$
\kappa_{\theta_{i} \theta_{j}}^{\left(\theta_{k}\right)}=\frac{\partial}{\partial \theta_{k}} \kappa_{\theta_{i} \theta_{j}},
$$

for $i, j, k=1,2, \ldots, p$.

\section{B. Cox-Snell Corrected ML Estimator}

Let $\hat{\theta}_{i}, i=1,2, \ldots, p$, be the ML estimator for $\theta_{i}$. Considering the mathematical expressions described in the previous subsection, the Cox-Snell expression for the bias of $\hat{\theta}_{i}$ is described here. Such bias correction was given an alternative form in terms of the cumulants of the log-likelihood derivatives [9]. It was established that the bias of $\hat{\theta}_{i}$ possesses the following formulation [9]:

$$
B\left(\widehat{\theta}_{i}\right)=\mathrm{E}\left(\widehat{\theta}_{i}\right)-\theta_{i}=\sum_{r, s, t} \kappa^{\theta_{i}, \theta_{r}} \kappa^{\theta_{s}, \theta_{t}}\left(\kappa_{\theta_{r} \theta_{s}}^{\left(\theta_{t}\right)}-\frac{1}{2} \kappa_{\theta_{r} \theta_{s} \theta_{t}}\right),
$$

Therefore, the corrected ML estimator $\widetilde{\theta}_{i}$ is defined by

$$
\widetilde{\theta}_{i}=\widehat{\theta}_{i}-\widehat{B}\left(\widehat{\theta}_{i}\right),
$$

where $\widehat{B}\left(\widehat{\theta}_{i}\right)$ represents the bias $B\left(\widehat{\theta}_{i}\right)$ evaluated not at $\theta_{i}$ but at $\widehat{\theta}_{i}$. Additionally, it was previously shown that (i) $\mathrm{E}\left[\widehat{B}\left(\widehat{\theta}_{i}\right)\right]=$ 
$O\left(N^{-2}\right)$, (ii) $\mathrm{E}\left(\widehat{\theta}_{i}\right)=\theta_{i}+O\left(N^{-1}\right)$, and (iii) $\mathrm{E}\left(\widetilde{\theta}_{i}\right)=$ $\theta_{i}+O\left(N^{-2}\right)$ [10]. Therefore the bias of $\tilde{\theta}_{i}$ has order of $N^{-2}$. In contrast, we have that $B\left(\widehat{\theta}_{i}\right)=O\left(N^{-1}\right)$ [8]. Thus, $\widetilde{\theta}_{i}$ is expected to possess better asymptotic properties when compared to $\widehat{\theta}_{i}$.

\section{Barndorff-Nielsen ML Estimator}

In [15] Barndorff-Nielsen introduced an improved ML estimation based on the modified profile likelihood. This method was previously employed in [16]-[18]. Below we furnish a brief outline of the technique.

Let the parameter vector $\boldsymbol{\theta}$ be split into two vectors such that $\boldsymbol{\theta}=\left[\begin{array}{ll}\boldsymbol{\psi}^{\top} & \boldsymbol{\lambda}^{\top}\end{array}\right]^{\top}$, where $\boldsymbol{\psi}$ and $\boldsymbol{\lambda}$ have $q$ and $p-q$ elements, respectively. Vector $\boldsymbol{\psi}$ is the interest parameter vector, whereas vector $\boldsymbol{\lambda}$ is the nuisance parameter vector. In [29], [30], the statistical inference of $\boldsymbol{\psi}$, for a known $\lambda$, was addressed. However, such calculation requires the marginal or conditional likelihood functions, which are often mathematically intractable [18].

A common solution to this conundrum is to approximate these functions by the profile log-likelihood [14], which is denoted by

$$
\tilde{\ell}(\boldsymbol{\psi})=\ell\left(\left[\begin{array}{c}
\psi \\
\widehat{\boldsymbol{\lambda}}_{\psi}
\end{array}\right]\right)
$$

where $\widehat{\lambda}_{\psi}$ is the maximum likelihood estimator of $\boldsymbol{\lambda}$ expressed in terms of the elements of $\boldsymbol{\psi}$.

However, such approximation may be problematic. In fact, the profile log-likelihood is not a bona fide log-likelihood function [15]. Moreover, the associated profile score and the information biases are only guaranteed to be $O(1)$ [31]. Therefore ML estimators based on profile log-likelihood are prone to bias issues, specially for small sample sizes [18].

To address this issue, several modifications for the profile log-likelihood function have been proposed [15], [29], [30], [32]. In the current work, we adopted the Barndorff-Nielsen modification [15], which is analytically tractable when the scaled complex Wishart distribution is considered.

Barndorff-Nielsen approximation for the marginal loglikelihood function is expressed according to [33]:

$$
\tilde{\ell}_{\mathrm{BN}}(\boldsymbol{\psi})=\tilde{\ell}(\boldsymbol{\psi})-\log \left|\frac{\partial \widehat{\boldsymbol{\lambda}}_{\psi}}{\partial \widehat{\boldsymbol{\lambda}}}\right|-\frac{1}{2} \log \left|\mathcal{J}_{\boldsymbol{\lambda} \boldsymbol{\lambda}}\left(\left[\begin{array}{c}
\boldsymbol{\psi} \\
\widehat{\boldsymbol{\lambda}}_{\psi}
\end{array}\right]\right)\right|,
$$

where $\hat{\lambda}$ is the unrestricted maximum likelihood estimators for the nuisance parameter $\boldsymbol{\lambda}$, and $\mathcal{J}_{\boldsymbol{\lambda} \boldsymbol{\lambda}}(\cdot)$ is the observed information matrix for $\boldsymbol{\lambda}$ given by:

$$
\mathcal{J}_{\boldsymbol{\lambda} \boldsymbol{\lambda}}\left(\left[\begin{array}{c}
\boldsymbol{\psi} \\
\hat{\boldsymbol{\lambda}}_{\psi}
\end{array}\right]\right)=-\frac{\partial^{2}}{\partial \boldsymbol{\lambda} \partial \boldsymbol{\lambda}^{\mathrm{H}}} \ell(\boldsymbol{\theta})
$$

The associated bias for $\widetilde{\ell}_{\mathrm{BN}}(\psi)$ is $O\left(N^{-1}\right)$ [33]. If $\widehat{\boldsymbol{\lambda}}_{\psi}=\widehat{\boldsymbol{\lambda}}$ for all $\psi$ (i.e., $\partial \widehat{\boldsymbol{\lambda}}_{\psi} / \partial \widehat{\boldsymbol{\lambda}}=\boldsymbol{I}_{p-q}$, where $\boldsymbol{I}_{a}$ represents a identity matrix with order $a$ ), (5) collapses into:

$$
\widetilde{\ell}_{\mathrm{BN}}(\boldsymbol{\psi})=\tilde{\ell}(\boldsymbol{\psi})-\frac{1}{2} \log \left|\mathcal{J}_{\lambda \lambda}\left(\left[\begin{array}{c}
\boldsymbol{\psi} \\
\hat{\boldsymbol{\lambda}}_{\psi}
\end{array}\right]\right)\right|
$$

Above expression is known as the Cox-Reid modified profile log-likelihood [29]. The vector $\widehat{\psi}_{\mathrm{BN}}$ that maximizes (5) is the Barndorff-Nielsen ML estimator for $\boldsymbol{\psi}$ [18].

It is shown in [33] that the bias of $\widehat{\psi}_{\mathrm{BN}}$ is $O\left(N^{-3 / 2}\right)$. Therefore, for this particular log-likelihood function, the $\mathrm{ML}$ estimator bias is also $O\left(N^{-3 / 2}\right)$. This is an improvement over the standard ML estimator, whose bias is $O\left(N^{-1}\right)$.

\section{IMPROVED ML ESTIMATION FOR THE SCALED COMPLEX WISHART DISTRIBUTION}

In this section we propose improvements to ML estimators for the parameters of the scaled complex Wishart distribution. We consider the methodologies described in the previous section, namely the Cox-Snell corrected ML estimation and the Barndorff-Nielsen ML estimation based on the modified profile log-likelihood function.

Mainly, our goal is to derive corrected ML estimators for $\boldsymbol{\Sigma}$ and $L$. However, as established in [23], we notice that $\mathrm{E}\left(\widehat{\boldsymbol{\Sigma}}_{\mathrm{ML}}\right)=\boldsymbol{\Sigma}$. Therefore, $\widehat{\boldsymbol{\Sigma}}_{\mathrm{ML}}$ is already unbiased, requiring no correction. Thus, we focus our efforts on obtaining a corrected ML estimator for $L$.

\section{A. Cox-Snell Corrected ML estimators for $\boldsymbol{\Sigma}$ and $L$}

According to (4), the Cox-Snell method yields an improved ML estimator for $L$, termed by $\widehat{L}_{\mathrm{IML}}$, given by

$$
\widehat{L}_{\mathrm{IML}}=\widehat{L}_{\mathrm{ML}}-\widehat{B}\left(\widehat{L}_{\mathrm{ML}}\right) \text {. }
$$

Now we aim at computing $\widehat{B}\left(\widehat{L}_{\mathrm{ML}}\right)$. For such firstly we need to derive $B\left(\widehat{L}_{\mathrm{ML}}\right)$. Invoking (3), we have that

$$
\begin{aligned}
B\left(\widehat{L}_{\mathrm{ML}}\right)= & \kappa^{L, L} \kappa^{L, L}\left(\kappa_{L L}^{(L)}-\frac{1}{2} \kappa_{L L L}\right) \\
& +\sum_{i=1}^{m^{2}} \sum_{j=1}^{m^{2}} \kappa^{L, L} \kappa^{\sigma_{i}, \sigma_{j}}\left(\kappa_{L \sigma_{i}}^{\left(\sigma_{i}\right)}-\frac{1}{2} \kappa_{L \sigma_{i} \sigma_{j}}\right) .
\end{aligned}
$$

Following algebraic manipulations, as detailed in Appendix A, we could simplify (8) and re-cast it as

$$
B(L)=\frac{m^{2}}{2 N L\left[\psi_{m}^{(1)}(L)-\frac{m}{L}\right]}-\frac{\frac{m}{2 L}+\psi_{m}^{(2)}(L)}{2 N\left[\psi_{m}^{(1)}(L)-\frac{m}{L}\right]^{2}} .
$$

Now replacing $L$ by $\widehat{L}_{\mathrm{ML}}$, we obtain $\widehat{B}\left(\widehat{L}_{\mathrm{ML}}\right)$. This quantity must be inserted back into (7) to furnish the proposed improved ML estimator for $L$.

\section{B. Modified profile log-likelihood for number of looks}

Following the discussed Barndorff-Nielsen technique, we derive the profile log-likelihood function associated to the scaled complex Wishart distribution. This quantity can be obtained by replacing the covariance matrix $\Sigma$ by its ML estimator $\widehat{\Sigma}_{\mathrm{ML}}$ in (1). This particular manipulation yields $\widetilde{\ell}(\cdot)$ 
as a function of the sought parameter $L$ :

$$
\begin{aligned}
\widetilde{\ell}(L)= & m N L(\log L-1)+(L-m) \sum_{k=1}^{N} \log \left|\boldsymbol{Z}_{k}\right| \\
& -N L \log |\overline{\boldsymbol{Z}}|-N \frac{m(m-1)}{2} \log \pi \\
& -N \sum_{k=0}^{m-1} \log \Gamma(L-k) .
\end{aligned}
$$

Thus, the profile score function is given by

$$
\begin{aligned}
\widetilde{U}(L)=\frac{\partial \widetilde{\ell}(L)}{\partial L}= & m N \log L-N \psi_{m}^{(0)}(L)-N \log |\overline{\boldsymbol{Z}}| \\
& +\sum_{k=1}^{N} \log \left|\boldsymbol{Z}_{k}\right| .
\end{aligned}
$$

From (1), we could express the profile score function in terms of $\boldsymbol{\sigma}=\operatorname{vec}(\boldsymbol{\Sigma})$, yielding:

$$
U(\boldsymbol{\sigma})=N \operatorname{vec}\left(\boldsymbol{\Sigma}^{-1} \overline{\boldsymbol{Z}} \boldsymbol{\Sigma}^{-1}-\boldsymbol{\Sigma}^{-1}\right) .
$$

By imposing $U(\boldsymbol{\sigma})=\mathbf{0}$, where $\mathbf{0}$ is a column vector of zeros, and solving the resulting system of equations, one obtains that the unrestricted and restricted ML estimators for $\sigma$ are given by $\operatorname{vec}(\overline{\boldsymbol{Z}})$. Moreover, we have that

$$
\frac{\partial \widehat{\boldsymbol{\sigma}}_{L}}{\partial \widehat{\boldsymbol{\sigma}}}=\boldsymbol{I}_{m^{2}},
$$

where $\widehat{\sigma}_{L}$ represents the restricted ML estimator. As a consequence, we are now in a position to use (6).

However, the following quantity is necessary:

$$
-\frac{1}{2} \log \left|\mathcal{J}_{\boldsymbol{\sigma} \sigma}\left(\left[\begin{array}{l}
L \\
\widehat{\sigma}
\end{array}\right]\right)\right|
$$

where $\widehat{\sigma}=\operatorname{vec}\left(\widehat{\boldsymbol{\Sigma}}_{\mathrm{ML}}\right)$. Due to its length, the mathematical derivation for this quantity is detailed in Appendix B. Thus, from (6), the modified profile log-likelihood according to the Barndorff-Nielsen technique is furnished by

$$
\tilde{\ell}_{\mathrm{BN}}(L)=\widetilde{\ell}(L)-\frac{m^{2}}{2}(\log N+\log L)-m \log \left|\overline{\boldsymbol{Z}}^{-1}\right| .
$$

As a consequence, the associated relative score function is expressed by

$$
\widetilde{U}_{\mathrm{BN}}(L)=\frac{\partial}{\partial L} \widetilde{\ell}_{\mathrm{BN}}(L)=\widetilde{U}(L)-\frac{m^{2}}{2 L} .
$$

As required, the sought estimator $\widehat{L}_{\mathrm{BN}}$ must satisfy $\widetilde{U}_{\mathrm{BN}}\left(\widehat{L}_{\mathrm{BN}}\right)=0$. In other words, it is the solution of the following non-linear equation:

$$
\begin{aligned}
m N \log \widehat{L}_{\mathrm{BN}} & -N \psi_{m}^{(0)}\left(\widehat{L}_{\mathrm{BN}}\right)-N \log |\overline{\boldsymbol{Z}}| \\
& +\sum_{k=1}^{N} \log \left|\boldsymbol{Z}_{k}\right|-\frac{m^{2}}{2 \widehat{L}_{\mathrm{BN}}}=0 .
\end{aligned}
$$

A closed-form expression for $\widehat{L}_{\mathrm{BN}}$ could not be given. Then we resort to numerical techniques, such as the Newton-Raphson method, as a means for solving (11).

\section{Performance and Assessment}

To assess the performance of the proposed estimators for the equivalent number of looks, we considered a simulation study on synthetic data based on Monte Carlo experiments. Such simulation included the following estimators:

1) the standard ML estimator $\left(\widehat{L}_{\mathrm{ML}}\right)[5]$;

2) the first and second trace moment estimators $\left(\widehat{L}_{\mathrm{MM} 1}\right.$ and $\widehat{L}_{\mathrm{MM} 2}$, respectively) [5];

3) the proposed ML estimator based on the Cox and Snell methodology $\left(\widehat{L}_{\mathrm{IML}}\right)$; and

4) the proposed ML estimator according to the BarndorffNielsen adjustment $\left(\widehat{L}_{\mathrm{BN}}\right)$.

The first three above-mentioned estimators were derived by Anfinsen et al. [5].

Simulation results, were assessed in terms of three statistical measures: (i) the mean squared error (MSE); (ii) the coefficient of variation $(\mathrm{CV})$; and (iii) the mean estimated values; and (iv) the bias curves $(B)$.

Subsequently we separate the best estimator among those proposed by Anfinsen et al. as indicated by the simulation study. Then such selected estimator as well as the proposed estimators are submitted to actual data analysis aiming at performance assessment.

\section{A. Simulation study}

The suggested Monte Carlo simulation employed 5500 replications as discussed in [34]. Additionally, the following simulation parameters were considered: (i) sample sizes $N \in\{9,49,121\}$; (ii) number of looks $L \in\{4,6,8,12\}$; and (iii) a covariance matrix given by

$$
\boldsymbol{\Sigma}_{0}=\left[\begin{array}{ccc}
962892 & 19171-3579 \mathbf{i} & -154638+191388 \mathbf{i} \\
& 56707 & -5798+16812 \mathbf{i} \\
& & 472251
\end{array}\right],
$$

where the omitted elements can be obtained from the conjugation of their respective symmetric elements.

The adopted sample sizes are associated to square windows of $\{3,7,11\}$ pixels, respectively. It is worth mentioning that higher values of $L$ represent images less affected by speckle noise. This particular estimated covariance matrix $\boldsymbol{\Sigma}_{0}$ was obtained by the E-SAR sensor over Weßling, Germany. In particular, it was employed in [35] for the characterization of urban areas.

The results for MSE, CV, and mean estimated values are shown in Table I. Initially we notice that the proposed estimators $\widehat{L}_{\mathrm{BN}}$ and $\widehat{L}_{\mathrm{IML}}$ could offer more accurate average ML estimates for the ENL when compared to the results derived from the other considered methods. In general terms, the increasing of sample size reduces the MSE and CV. This behavior is expected, because the estimators are asymptotically unbiased. Results also show that the estimator $\widehat{L}_{\mathrm{IML}}$ presented the best performance in terms of MSE and CV in all considered cases. This last result provides even stronger evidence in favor of the accuracy of $\widehat{L}_{\mathrm{IML}}$. This is because bias corrected estimators do not always yield better procedures than ML estimators. Indeed, bias corrected estimators can offer 
larger variance values, leading to increased MSE values when compared to uncorrected methods [9].

Fig. 1 presents the values of bias for several sample sizes. The following inequality is verified:

$$
\widehat{B}\left(\widehat{L}_{\mathrm{MM} 1}\right) \geq \widehat{B}\left(\widehat{L}_{\mathrm{MM} 2}\right) \geq \widehat{B}\left(\widehat{L}_{\mathrm{ML}}\right) \geq \widehat{B}\left(\widehat{L}_{\mathrm{BN}}\right) \geq \widehat{B}\left(\widehat{L}_{\mathrm{IML}}\right) .
$$

It is noteworthy that, with the exception of $\widehat{L}_{\mathrm{IML}}$, all the procedures overestimate the equivalent number of looks, i.e., they lead to decisions that assume that there is more information in the data than the true content. The only estimator with a different behavior is $\widehat{L}_{\mathrm{IML}}$. It consistently exhibits the smallest bias, which is reduced with increasing number of looks. For large $L$ and small samples, its bias becomes negative. The other estimators have their biases increased with the number of looks.

The estimator $\widehat{L}_{\text {IML }}$ was also less affected by the increasing of number of looks. Thus, this estimator performed well enduring both variations of the sample size as well as the number of looks. We also emphasize that the better performance of estimator $\widehat{L}_{\mathrm{ML}}$ in comparison with $\widehat{L}_{\mathrm{MM} 1}$ and $\widehat{L}_{\mathrm{MM} 2}$ is in agreement with the findings of Anfisen et al. [5]. However, when considering the proposed estimators, we identify that $\widehat{L}_{\mathrm{ML}}, \widehat{L}_{\mathrm{IML}}$, and $\widehat{L}_{\mathrm{BN}}$ as the more accurate estimators among the considered techniques.

\section{B. Analysis with actual data}

Now we aim at submitting actual PolSAR data to the three best estimators separated in the previous subsection. We adopted the San Francisco AIRSAR image as a source of actual data. This classical multilook PolSAR image was obtained by the AIRSAR sensor operating at $\mathrm{L}$ band with four nominal looks [36]. Fig. 2 presents the $\mathrm{HH}$ channel intensity data of the $150 \times 150$ San Francisco image. Subareas were selected from the main image. By means of visual inspection, three categories were sought: (i) ocean; (ii) forest; and (iii) urban area.

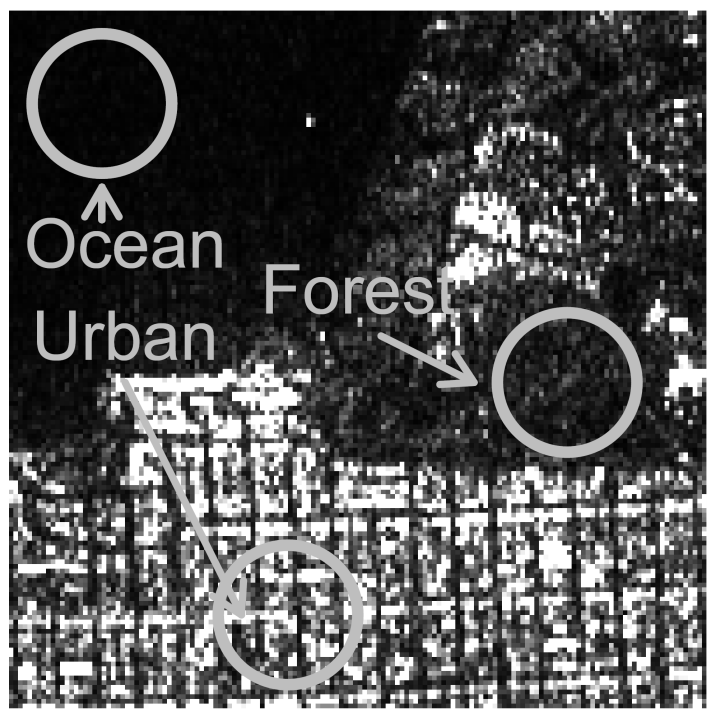

Fig. 2. San Francisco image with selected regions.
For each selected subregion, we considered 5500 subsamples without replacement with size $N \in\{9,36,121,144\}$. These subsamples are then submitted to parameter estimation.

Table II displays the obtained results. For the selected ocean subregion, the corrected estimator $\widehat{L}_{\text {IML }}$ presented the smallest values of MSE and CV. Thus, the furnished estimates were more accurate. As discussed by Anfinsen et al. [5], textured regions yield to underestimation effects. Then correction schemes tend to become less efficient when compared to usual ML estimation $\widehat{L}_{\mathrm{ML}}$.

Indeed, as reported in [2], heavily textured images, such as forest and urban, are less adequately described by the complex Wishart distribution. This phenomenon was also verified in [37]-[39]. Therefore, the proposed tools are expected to excel at low textured samples.

Fig. 3 shows the bias curves for the ocean region in which case the proposed tools were superior. These curves indicate that the estimator $\widehat{L}_{\mathrm{IML}}$ could outperform the usual ML estimator when homogeneous targets are considered.

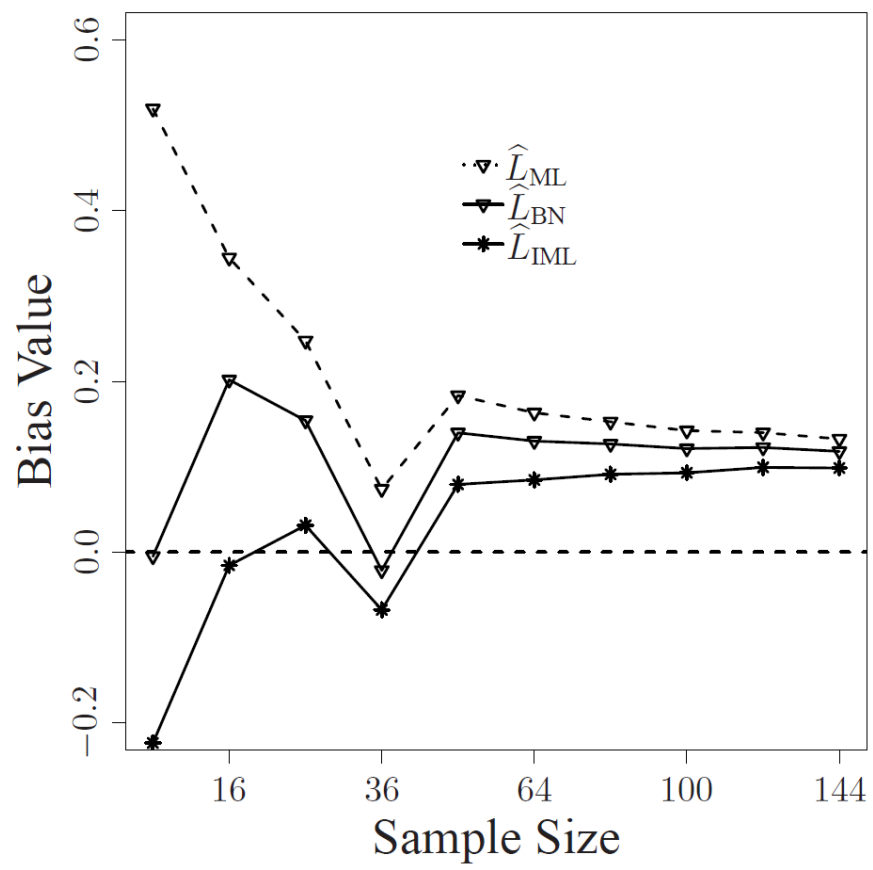

Fig. 3. Bias curves in terms of sample sizes for actual data.

Finally, we notice that both Table II and Figure 3 indicate that the discussed estimators possess similar asymptotic properties. This behavior is expected and can derived from theoretical results as shown in [7], [40].

\section{CONCLUSION}

In this paper, we proposed two new improved corrected ML estimators for the scaled complex Wishart distribution. Emphasis was put on the estimation of the number of looks. In particular, we introduced closed-form expressions for the bias and for the modified profile likelihood according to methodologies advanced by Cox and Snell [10] and BarndorffNielsen [15]. 


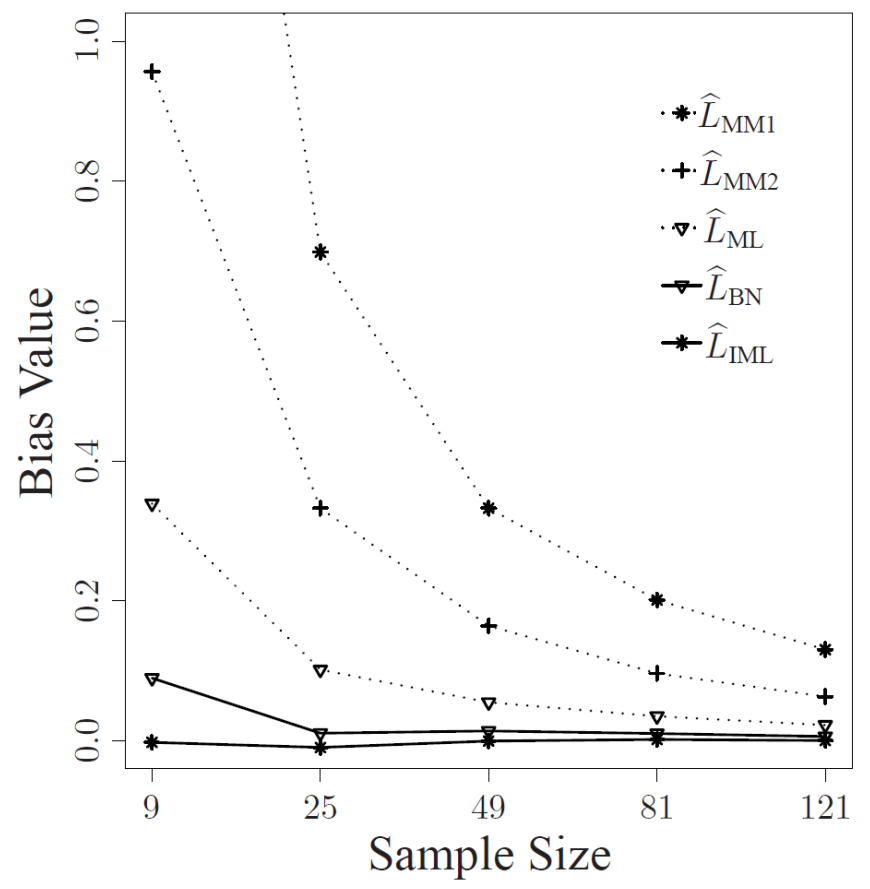

(a) $L=4$

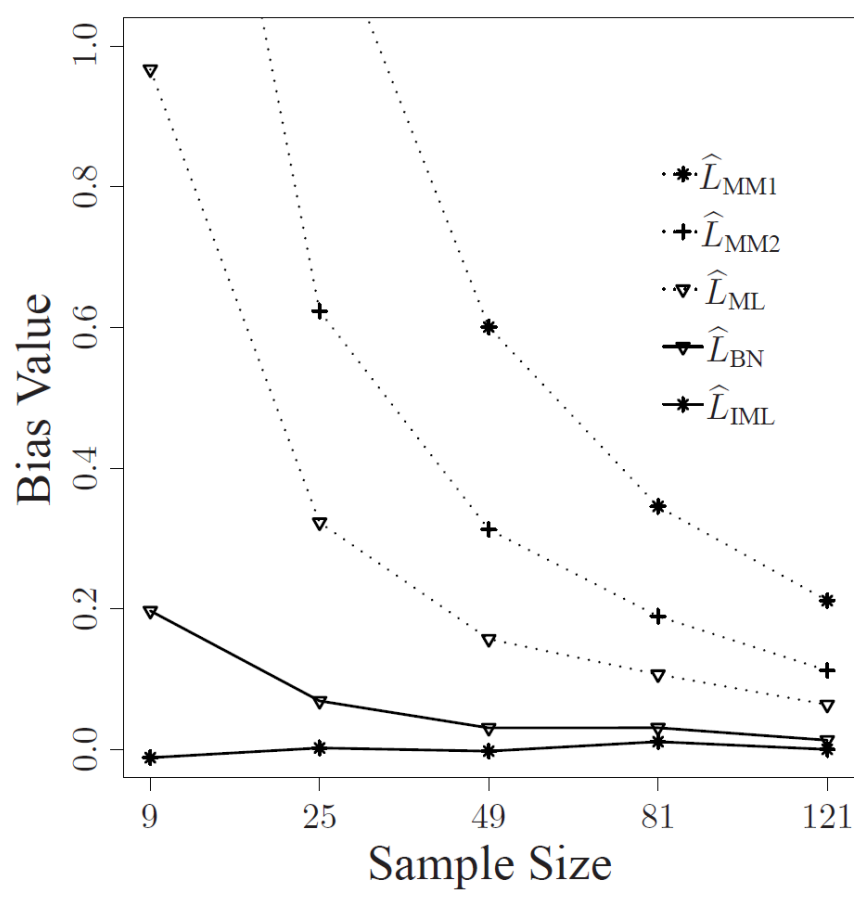

(c) $L=8$

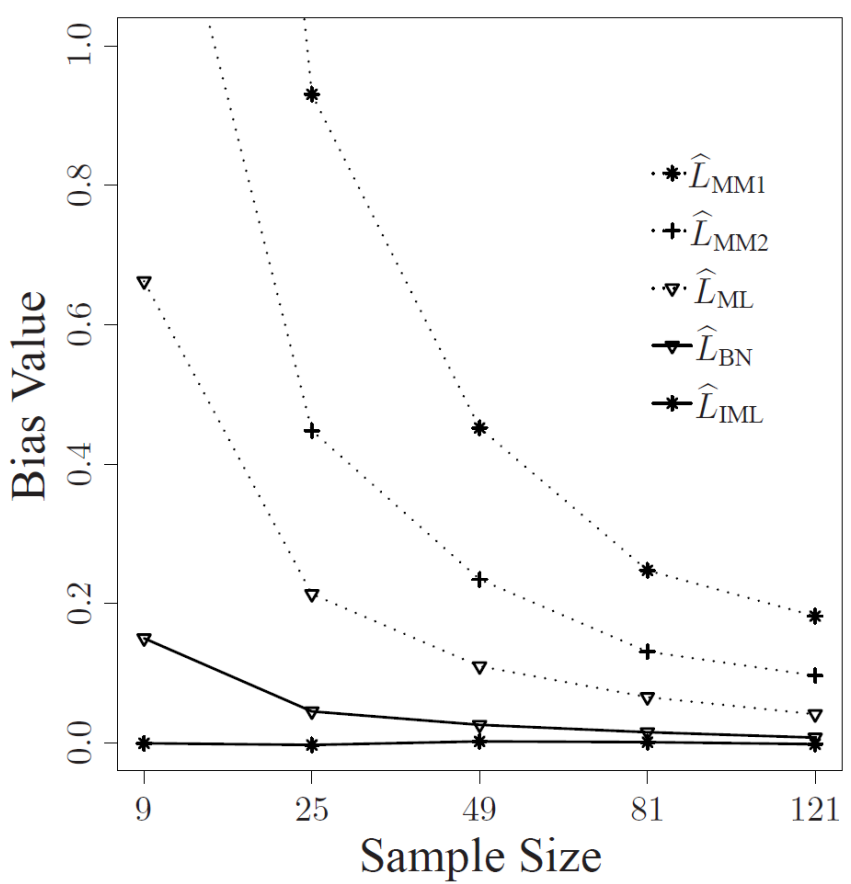

(b) $L=6$

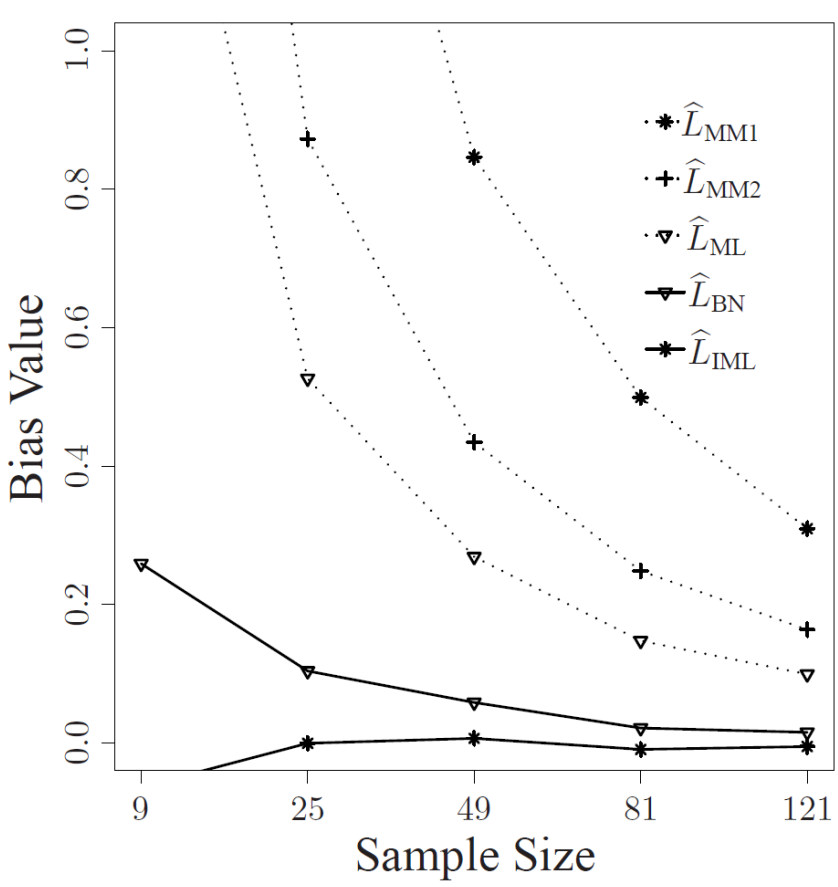

(d) $L=12$

Fig. 1. Bias curves in terms of sample sizes for synthetic data. 
TABLE I

MEASURES OF THE ESTIMATORS PERFORMANCE WITH SYNTHETIC DATA

\begin{tabular}{|c|c|c|c|c|c|c|c|c|c|c|c|c|c|}
\hline \multirow[b]{2}{*}{$\widehat{L}$} & \multirow[b]{2}{*}{$N$} & \multicolumn{3}{|c|}{$L=4$} & \multicolumn{3}{|c|}{$L=6$} & \multicolumn{3}{|c|}{$L=8$} & \multicolumn{3}{|c|}{$L=12$} \\
\hline & & $\operatorname{mean}(\widehat{L})$ & MSE & $\mathrm{CV}$ & $\operatorname{mean}(\widehat{L})$ & MSE & $\mathrm{CV}$ & $\operatorname{mean}(\widehat{L})$ & MSE & $\mathrm{CV}$ & $\operatorname{mean}(\widehat{L})$ & MSE & $\mathrm{CV}$ \\
\hline$\widehat{L}_{\mathrm{ML}}$ & \multirow[t]{5}{*}{9} & 4.339 & \multicolumn{2}{|c|}{0.4140 .126} & 6.663 & \multicolumn{2}{|c|}{1.3730 .145} & 8.967 & \multicolumn{2}{|c|}{2.8100 .153} & 13.538 & \multicolumn{2}{|c|}{6.9630 .158} \\
\hline$\widehat{L}_{\mathrm{MM} 1}$ & & 6.278 & \multicolumn{2}{|c|}{23.1740 .676} & 9.344 & 52.605 & 0.689 & 12.478 & \multicolumn{2}{|c|}{95.9780 .698} & 18.119 & \multicolumn{2}{|c|}{190.4320 .68} \\
\hline$\widehat{L}_{\mathrm{MM} 2}$ & & 4.957 & \multicolumn{2}{|c|}{2.9930 .291} & 7.401 & 6.399 & 0.285 & 9.849 & \multicolumn{2}{|c|}{11.8000 .294} & 14.606 & \multicolumn{2}{|c|}{$\begin{array}{lll}6 & 24.977 & 0.292\end{array}$} \\
\hline$\widehat{L}_{\mathrm{IML}}$ & & 3.998 & \multicolumn{2}{|c|}{0.2210 .118} & 6.000 & 0.695 & 0.139 & 7.989 & \multicolumn{2}{|c|}{1.3980 .148} & 11.937 & \multicolumn{2}{|c|}{3.4350 .155} \\
\hline$\widehat{L}_{\mathrm{BN}}$ & & 4.090 & \multicolumn{2}{|c|}{0.2430 .118} & 6.150 & 0.760 & 0.140 & 8.197 & \multicolumn{2}{|c|}{1.5180 .148} & 12.259 & \multicolumn{2}{|l|}{3.700} \\
\hline \multirow{5}{*}{\multicolumn{2}{|c|}{49}} & 4.055 & \multicolumn{2}{|c|}{0.0420 .049} & 6.110 & 0.133 & 0.057 & 8.157 & \multicolumn{2}{|c|}{0.2580 .059} & 12.269 & \multicolumn{2}{|c|}{0.6610 .063} \\
\hline & & 4.333 & \multicolumn{2}{|c|}{$\begin{array}{ll}1.045 & 0.223\end{array}$} & 6.452 & 2.201 & 0.219 & 8.601 & \multicolumn{2}{|c|}{3.8090 .216} & 12.847 & \multicolumn{2}{|c|}{$8.363 \quad 0.215$} \\
\hline & & 4.165 & \multicolumn{2}{|c|}{0.2940 .124} & 6.235 & 0.633 & 0.122 & 8.313 & \multicolumn{2}{|c|}{1.0930 .120} & 12.435 & \multirow{2}{*}{$\begin{array}{l}2.388 \\
0.559\end{array}$} & 0.119 \\
\hline & & 4.000 & \multicolumn{2}{|c|}{0.0370 .048} & 6.002 & 0.115 & 0.056 & 7.998 & 0.222 & 0.059 & 12.007 & & 0.062 \\
\hline & & 4.014 & 0.037 & 0.048 & 6.026 & 0.117 & 0.057 & 8.031 & 0.225 & 0.059 & 12.059 & 0.568 & 0.062 \\
\hline & 121 & 4.023 & 0.01 & 0.0 & 6.041 & 0.048 & 0.0 & 8.064 & 0.096 & 0.0 & 12.100 & 0.237 & 0.039 \\
\hline & & 4.131 & 0.350 & 0.140 & 6.182 & 0.738 & 0.136 & 8.212 & 1.261 & 0.1 & 12.310 & 2.820 & 0.134 \\
\hline & & 4.063 & 0.108 & 0.080 & 6.097 & 0.233 & 0.077 & 8.113 & 0.403 & 0.077 & 12.164 & 0.871 & 0.076 \\
\hline & & 4.001 & 0.015 & 0.031 & 5.998 & 0.045 & 0.035 & 8.001 & 0.090 & 0.038 & 11.995 & 0.222 & 0.039 \\
\hline & & 4.006 & 0.015 & 0.031 & 6.008 & 0.045 & 0.035 & 8.014 & 0.091 & 0.038 & 12.016 & 0.223 & 0.039 \\
\hline
\end{tabular}

TABLE II

MEASURES OF THE ESTIMATORS PERFORMANCE WITH ACTUAL DATA

\begin{tabular}{|c|c|c|c|c|c|c|c|c|c|c|}
\hline \multirow[b]{2}{*}{ Regions } & \multirow[b]{2}{*}{$N$} & \multicolumn{3}{|c|}{$\widehat{L}_{\mathrm{ML}}$} & \multicolumn{3}{|c|}{$\widehat{L}_{\mathrm{IML}}$} & \multicolumn{3}{|c|}{$\widehat{L}_{\mathrm{BN}}$} \\
\hline & & $\operatorname{mean}(\widehat{L})$ & MSE & $\mathrm{CV}$ & $\operatorname{mean}(\widehat{L})$ & MSE & $\mathrm{CV}$ & $\operatorname{mean}(\widehat{L})$ & MSE & $\mathrm{CV}$ \\
\hline \multirow[t]{4}{*}{ Ocean } & 9 & 4.520 & 0.747 & 0.153 & 3.776 & 0.350 & 0.145 & 3.995 & 1.376 & 0.294 \\
\hline & 36 & 4.074 & 0.623 & 0.193 & 3.932 & 0.582 & 0.193 & 3.979 & 0.739 & 0.216 \\
\hline & 121 & 4.140 & 0.034 & 0.029 & 4.099 & 0.024 & 0.029 & 4.123 & 0.029 & 0.029 \\
\hline & 144 & 4.133 & 0.029 & 0.026 & 4.099 & 0.021 & 0.025 & 4.118 & 0.025 & 0.026 \\
\hline \multirow{4}{*}{\multicolumn{2}{|c|}{ Forest }} & 3.293 & 0.599 & 0.095 & 3.069 & 0.938 & 0.087 & 3.159 & 0.792 & 0.092 \\
\hline & & 3.119 & 0.794 & 0.043 & 3.075 & 0.872 & 0.042 & 3.089 & 0.852 & 0.049 \\
\hline & & 2.809 & 2.041 & 0.281 & 2.798 & 2.065 & 0.281 & 2.818 & 1.987 & 0.273 \\
\hline & & 3.082 & 0.845 & 0.017 & 3.072 & 0.864 & 0.017 & 3.075 & 0.858 & 0.017 \\
\hline \multirow{4}{*}{\multicolumn{2}{|c|}{ Urban }} & 2.738 & 2.439 & 0.336 & 2.492 & 3.239 & 0.394 & 2.678 & 2.451 & 0.313 \\
\hline & & 2.567 & 2.486 & 0.256 & 2.529 & 2.594 & 0.260 & 2.454 & 2.989 & 0.316 \\
\hline & & 2.218 & 3.959 & 0.399 & 2.209 & 3.990 & 0.401 & 2.177 & 4.146 & 0.417 \\
\hline & & 2.447 & 2.833 & 0.265 & 2.439 & 2.857 & 0.265 & 2.426 & 2.923 & 0.275 \\
\hline
\end{tabular}

By means of Monte Carlo simulations, the proposed techniques were assessed and compared with estimators presented by Anfinsen et al. [5]. Adopted figures of merit include MSE, coefficient of variation, mean of estimated values, and the bias curves. Results showed that the proposed corrected estimators outperform all considered estimators. Actual data were also analyzed revealing that the proposed estimators are superior when homogeneous scenes are considered.

\section{APPENDIX A}

\section{Cumulants FOR THE COX-SNEll CORRECTION}

In this appendix, we present closed-form expressions for (i) some cumulants from the scaled complex Wishart distribution as well as (ii) the second-order bias according to the Cox-Snell approach.

From (1), the second and third-order derivatives are given by

$$
\begin{aligned}
U_{L L}= & \frac{N m}{L}-N \psi_{m}^{(1)}(L) \\
U_{L \sigma_{i}}= & N\left[\operatorname{vec}\left(\boldsymbol{\Sigma}^{-1} \overline{\boldsymbol{Z}} \boldsymbol{\Sigma}^{-1}-\boldsymbol{\Sigma}^{-1}\right)\right]_{i} \\
U_{\sigma_{i} \sigma_{j}}= & L N\left[\left(\boldsymbol{\Sigma}^{-1} \otimes \boldsymbol{\Sigma}^{-1}\right)-\left(\boldsymbol{\Sigma}^{-1} \otimes \boldsymbol{\Sigma}^{-1}\right) \overline{\boldsymbol{Z}} \boldsymbol{\Sigma}^{-1}\right. \\
& \left.\quad-\left(\boldsymbol{\Sigma}^{-1} \otimes \boldsymbol{\Sigma}^{-1}\right) \boldsymbol{\Sigma}^{-1} \overline{\boldsymbol{Z}}\right]_{i, j} \\
U_{L \sigma_{i} \sigma_{j}}= & L^{-1} U_{\sigma_{i} \sigma_{j}} \\
U_{\sigma_{i} \sigma_{j} \sigma_{k}=} & L N\left[3\left(\boldsymbol{\Sigma}^{-1} \otimes \boldsymbol{\Sigma}^{-1}\right) \otimes\left(\boldsymbol{\Sigma}^{-1} \overline{\boldsymbol{Z}} \boldsymbol{\Sigma}^{-1}\right)\right. \\
& +3\left(\boldsymbol{\Sigma}^{-1} \otimes \boldsymbol{\Sigma}^{-1}\right) \otimes\left(\boldsymbol{\Sigma}^{-1} \boldsymbol{\Sigma}^{-1} \overline{\boldsymbol{Z}}\right) \\
& \left.-2\left(\boldsymbol{\Sigma}^{-1} \otimes \boldsymbol{\Sigma}^{-1}\right) \otimes \boldsymbol{\Sigma}^{-1}\right]_{k+(i-1) m^{2}, k+(j-1) m^{2}}
\end{aligned}
$$

for $i, k, j=1,2, \ldots, m^{2}$, where $\otimes$ is the Kronecker product and $\sigma_{i}$ is the $i$ th element of vector $\boldsymbol{\sigma}$.

Based on the above results, we derive the following expression for the cumulants: 


$$
\begin{aligned}
\kappa^{L, L}= & \frac{1}{N}\left[\psi_{m}^{(1)}(L)-\frac{m}{L}\right]^{-1} \\
\kappa^{L, \sigma_{i}}= & 0, \quad \kappa^{\sigma_{i}, \sigma_{j}}=\frac{1}{N L}(\boldsymbol{\Sigma} \otimes \boldsymbol{\Sigma})_{i, j} \\
\kappa_{L L L}= & \kappa_{L L}^{(L)}=-N\left[\frac{m}{L^{2}}+\psi_{m}^{(2)}(L)\right] \\
\kappa_{L L \sigma_{i}}= & \kappa_{L L}^{\left(\sigma_{i}\right)}=\kappa_{L \sigma_{i}}^{\left(\sigma_{j}\right)}=0, \\
\kappa_{\sigma_{i} \sigma_{j} L}= & -N\left(\boldsymbol{\Sigma}^{-1} \otimes \boldsymbol{\Sigma}^{-1}\right)_{i, j}, \\
\kappa_{\sigma_{i} \sigma_{j}}^{\left(\sigma_{k}\right)}= & 2 L N \\
& {\left[\left(\boldsymbol{\Sigma}^{-1} \otimes \boldsymbol{\Sigma}^{-1}\right) \otimes \boldsymbol{\Sigma}^{-1}\right]_{k+(i-1) m^{2}, k+(j-1) m^{2}}, } \\
\kappa_{\sigma_{i} \sigma_{j} \sigma_{k}}= & 4 L N\left[\left(\boldsymbol{\Sigma}^{-1} \otimes \boldsymbol{\Sigma}^{-1}\right) \otimes \boldsymbol{\Sigma}^{-1}\right]_{k+(i-1) m^{2}, k+(j-1) m^{2}} .
\end{aligned}
$$

Since $\kappa^{L \sigma_{i}}=0$, parameters $L$ and $\sigma_{i}$, for $i=1,2, \ldots, m^{2}$, are orthogonal [41]. Therefore, expression (3) yields:

$$
\begin{aligned}
B\left(\widehat{L}_{\mathrm{ML}}\right)= & \kappa^{L, L} \kappa^{L, L}\left(\kappa_{L L}^{(L)}-\frac{1}{2} \kappa_{L L L}\right) \\
& +\sum_{i=1}^{m^{2}} \sum_{j=1}^{m^{2}} \kappa^{L, L} \kappa^{\sigma_{i}, \sigma_{j}}\left(\kappa_{L \sigma_{i}}^{\left(\sigma_{i}\right)}-\frac{1}{2} \kappa_{L \sigma_{i} \sigma_{j}}\right) .
\end{aligned}
$$

Above formula can be given a closed form expression. Indeed, from (15), we have that

$$
\begin{aligned}
\kappa^{\sigma_{i}, \sigma_{j}}\left(\kappa_{L \sigma_{i}}^{\left(\sigma_{i}\right)}\right. & \left.-\frac{1}{2} \kappa_{L \sigma_{i} \sigma_{j}}\right) \\
& =\frac{1}{N L}(\boldsymbol{\Sigma} \otimes \boldsymbol{\Sigma})_{i, j}\left(0+\frac{N}{2}\left\{\boldsymbol{\Sigma}^{-1} \otimes \boldsymbol{\Sigma}^{-1}\right\}_{i, j}\right) \\
& =\frac{1}{2 L}(\boldsymbol{\Sigma} \otimes \boldsymbol{\Sigma})_{i, j}\left\{(\boldsymbol{\Sigma} \otimes \boldsymbol{\Sigma})^{-1}\right\}_{i, j} .
\end{aligned}
$$

Considering the general expression of the elements of the inverse matrix [42, pp. 82], we have:

$$
\sum_{i=1}^{m^{2}} \sum_{j=1}^{m^{2}}\{\boldsymbol{\Sigma} \otimes \boldsymbol{\Sigma}\}_{i, j}\left\{(\boldsymbol{\Sigma} \otimes \boldsymbol{\Sigma})^{-1}\right\}_{i, j}=m^{2} .
$$

Therefore, we obtain:

$$
B\left(\widehat{L}_{\mathrm{ML}}\right)=\frac{m^{2}}{2 N L\left[\psi_{m}^{(1)}(L)-\frac{m}{L}\right]}-\frac{\frac{m}{2 L}+\psi_{m}^{(2)}(L)}{2 N\left[\psi_{m}^{(1)}(L)-\frac{m}{L}\right]^{2}} .
$$

\section{APPENDIX B}

\section{DERIVATION FOR BARNDORFF-NIELSEN APPROXIMATION}

In the subsequent discussion, we aim to detail the derivation of the Barndorff-Nielsen approximation for the marginal loglikelihood function of the scaled complex Wishart distribution. In particular, this appendix addresses the derivation for the quantity $-\frac{1}{2} \log \left|\mathcal{J}_{\boldsymbol{\sigma} \sigma}\left(\left[\begin{array}{l}L \\ \widehat{\sigma}\end{array}\right]\right)\right|$ discussed in Subsection IV-B.

Based on results (12)-(14), one can rewrite the observed information matrix from the scaled complex Wishart distribution by the following matrix equation:

$$
\begin{gathered}
{\left[\begin{array}{cc}
U_{L L} & U_{L \operatorname{vec}(\boldsymbol{\Sigma})}^{\top} \\
U_{\operatorname{vec}(\boldsymbol{\Sigma})^{*} L} & U_{\operatorname{vec}(\boldsymbol{\Sigma}) \operatorname{vec}(\boldsymbol{\Sigma})^{*}}
\end{array}\right]=} \\
{\left[\begin{array}{cc}
\frac{m N}{L}-N \psi_{m}^{(1)}(L) & N \operatorname{vec}\left(\boldsymbol{\Sigma}^{-1} \overline{\boldsymbol{Z}} \boldsymbol{\Sigma}^{-1}-\boldsymbol{\Sigma}^{-1}\right)^{\top} \\
N \operatorname{vec}\left(\boldsymbol{\Sigma}^{-1} \overline{\boldsymbol{Z}} \boldsymbol{\Sigma}^{-1}-\boldsymbol{\Sigma}^{-1}\right)^{*} & U_{\operatorname{vec}(\boldsymbol{\Sigma}) \operatorname{vec}(\boldsymbol{\Sigma})^{*}}
\end{array}\right],}
\end{gathered}
$$

where $U_{L \operatorname{vec}(\boldsymbol{\Sigma})}^{\top}$, defined by

$$
U_{L \operatorname{vec}(\boldsymbol{\Sigma})}^{\top}=\frac{\partial^{2}}{\partial L \partial \operatorname{vec}(\boldsymbol{\Sigma})^{\top}} \ell(\boldsymbol{\theta}),
$$

is a vector whose the $i$ th-element is given in (13) and [5]

$$
\begin{aligned}
U_{\operatorname{vec}(\boldsymbol{\Sigma}) \operatorname{vec}(\boldsymbol{\Sigma})^{*}}= & \frac{\partial^{2}}{\partial \operatorname{vec}(\boldsymbol{\Sigma})^{*} \partial \operatorname{vec}(\boldsymbol{\Sigma})^{\top}} \ell(\boldsymbol{\theta}) \\
= & L N\left[\left(\boldsymbol{\Sigma}^{-1} \otimes \boldsymbol{\Sigma}^{-1}\right)-\left(\boldsymbol{\Sigma}^{-1} \otimes \boldsymbol{\Sigma}^{-1}\right) \overline{\boldsymbol{Z}} \boldsymbol{\Sigma}^{-1}\right. \\
& \left.-\left(\boldsymbol{\Sigma}^{-1} \otimes \boldsymbol{\Sigma}^{-1}\right) \boldsymbol{\Sigma}^{-1} \overline{\boldsymbol{Z}}\right] .
\end{aligned}
$$

Thus, the quantity $\mathcal{J}_{\sigma \sigma}\left(\left[\begin{array}{l}L \\ \widehat{\sigma}\end{array}\right]\right)$ can be defined by

$$
\begin{aligned}
\mathcal{J}_{\boldsymbol{\sigma} \boldsymbol{\sigma}}\left(\left[\begin{array}{l}
L \\
\widehat{\boldsymbol{\sigma}}
\end{array}\right]\right) & =-\left.U_{\operatorname{vec}(\boldsymbol{\Sigma}) \operatorname{vec}(\boldsymbol{\Sigma})^{*}}\right|_{\boldsymbol{\Sigma}=\overline{\boldsymbol{Z}}} \\
& =N L \overline{\boldsymbol{Z}}^{-1} \otimes \overline{\boldsymbol{Z}}^{-1} .
\end{aligned}
$$

and, therefore, the following quantity combined with profile log-likelihood (9) at (6) yields the Barndorff-Nielsen approximation for the marginal log-likelihood function:

$$
\begin{aligned}
-\frac{1}{2} \log & \left|\mathcal{J}_{\boldsymbol{\sigma} \boldsymbol{\sigma}}\left(\left[\begin{array}{l}
L \\
\widehat{\boldsymbol{\sigma}}
\end{array}\right]\right)\right| \\
& =-\frac{m^{2}}{2}(\log N+\log L)-\frac{1}{2} \log \left|\overline{\boldsymbol{Z}}^{-1} \otimes \overline{\boldsymbol{Z}}^{-1}\right| .
\end{aligned}
$$

From [43, result 11.1 (1), page 235], we have that

$$
\log \left|\overline{\boldsymbol{Z}}^{-1} \otimes \overline{\boldsymbol{Z}}^{-1}\right|=2 m \log \left|\overline{\boldsymbol{Z}}^{-1}\right| .
$$

Thus, (16) can be simplified according to:

$$
\begin{aligned}
-\frac{1}{2} \log \left|\mathcal{J}_{\boldsymbol{\sigma} \boldsymbol{\sigma}}\left(\left[\begin{array}{l}
L \\
\hat{\boldsymbol{\sigma}}
\end{array}\right]\right)\right| \\
=-\frac{m^{2}}{2}(\log N+\log L)-m \log \left|\overline{\boldsymbol{Z}}^{-1}\right| .
\end{aligned}
$$

Thus, we have that (10) holds true.

\section{REFERENCES}

[1] J. S. Lee and E. Pottier, Polarimetric Radar Imaging: From Basics to Applications. Boca Raton: CRC, 2009.

[2] C. C. Freitas, A. C. Frery, and A. H. Correia, "The polarimetric $G$ distribution for SAR data analysis," Environmetrics, vol. 16, no. 1, pp. 13-31, 2005.

[3] A. C. Frery, A. D. C. Nascimento, and R. J. Cintra, "Analytic expressions for stochastic distances between relaxed complex Wishart distributions," IEEE Transactions on Geoscience and Remote Sensing, in press-. [Online]. Available: http://tiny.tw/3aIN

[4] A. D. C. Nascimento, M. M. Horta, A. C. Frery, and R. J. Cintra, "Comparing edge detection methods based on stochastic entropies and distances for polsar imagery," IEEE Journal of Selected Topics in Applied Earth Observations and Remote Sensing, in press. [Online]. Available: http://tiny.tw/3aIP

[5] S. N. Anfinsen, A. P. Doulgeris, and T. Eltoft, "Estimation of the equivalent number of looks in polarimetric synthetic aperture radar imagery," IEEE Transactions on Geoscience and Remote Sensing, vol. 47, pp. 3795-3809, Nov. 2009.

[6] C. H. Gierull and I. C. Sikaneta, "Estimating the effective number of looks in interferometric SAR data," IEEE Transactions on Geoscience and Remote Sensing, vol. 40, pp. 1733-1742, Aug. 2002.

[7] G. Casella and R. L. Berger, Statistical Inference. Duxbury Press, 2002.

[8] K. L. P. Vasconcellos, A. C. Frery, and L. B. Silva, "Improving estimation in speckled imagery," Computational Statistics, vol. 20, no. 3, pp. 503-519, 2005. 
[9] G. M. Cordeiro, "Bias correction," in International Encyclopedia of Statistical Science, M. Lovric, Ed. Springer Berlin Heidelberg, 2011, pp. $148-152$.

[10] D. R. Cox and E. J. Snell, "A general definition of residuals," Journal of the Royal Statistical Society. Series B (Methodological), vol. 30, no. 2, pp. 248-275, 1968.

[11] A. C. Frery, H. J. Muller, C. C. F. Yanasse, and S. J. S. Sant'Anna, "A model for extremely heterogeneous clutter," IEEE Transactions on Geoscience and Remote Sensing, vol. 35, pp. 648-659, May 1997.

[12] D. M. Pianto and F. Cribari-Neto, "Dealing with monotone likelihood in a model for speckled data," Computational Statistics \& Data Analysis, vol. 55, no. 3, pp. $1394-1409,2011$

[13] T. A. Severini, Likelihood Methods in Statistics. Oxford University Press, 2000.

[14] D. A. S. Fraser and N. Reid, "Adjustments to profile likelihood," Biometrika, vol. 76, no. 3, pp. 477-488, 1989.

[15] O. E. Barndorff-Nielsen, "Adjusted versions of profile likelihood and directed likelihood, and extended likelihood," Journal of the Royal Statistical Society. Series B (Methodological), vol. 56, no. 1, pp. 125140, 1994.

[16] A. H. M. A. Cysneiros, F. Cribari-Neto, and C. A. G. Araújo Jr., "On Birnbaum-Saunders inference," Computational Statistics \& Data Analysis, vol. 52, no. 11, pp. 4939-4950, 2008.

[17] T. Melo and S. Ferrari, "A modified signed likelihood ratio test in elliptical structural models," AStA Advances in Statistical Analysis, vol. 94, pp. 75-87, 2010.

[18] M. Silva, F. Cribari-Neto, and A. C. Frery, "Improved likelihood inference for the roughness parameter of the GA0 distribution," Environmetrics, vol. 19 , no. 4, pp. 347-368, 2008.

[19] A. C. Frery, F. Cribari-Neto, and M. O. Souza, "Analysis of minute features in speckled imagery with maximum likelihood estimation," EURASIP Journal on Advances in Signal Processing, vol. 2004, no. 16 pp. 2476-2491, 2004.

[20] F. T. Ulaby and C. Elachi, Radar Polarimetriy for Geoscience Applications. Norwood: Artech House, 1990.

[21] A. Freeman and S. L. Durden, "A three-component scattering model for polarimetric SAR data," IEEE Transactions on Geoscience and Remote Sensing, vol. 36, pp. 963-973, May 1998.

[22] N. R. Goodman, "Statistical analysis based on a certain complex Gaussian distribution (an introduction)," The Annals of Mathematical Statistics, vol. 34, pp. 152-177, 1963.

[23] A. C. Frery, R. J. Cintra, and A. D. C. Nascimento, "Entropy-based statistical analysis of PolSAR data," IEEE Transactions on Geoscience and Remote Sensing, vol. 51, pp. 3733-3743, Jun. 2013.

[24] J. E. Gentle, Elements of Computational Statistics, ser. Statistics and computing. Springer, 2002.

[25] A. Restrepo and A. C. Bovik, "Adaptive trimmed mean filters for image restoration," IEEE Transactions on Acoustics, Speech and Signal Processing, vol. 36, pp. 1326 -1337, Aug. 1988.

[26] L. Torres, S. J. S. Sant'Anna, C. C. Freitas, and A. C. Frery, "Speckle reduction in polarimetric SAR imagery with stochastic distances and nonlocal means," Pattern Recognition, in press. [Online]. Available: http://tiny.tw/3aIE

[27] C. Oliver and S. Quegan, Understanding Synthetic Aperture Radar Images, ser. The SciTech Radar and Defense series. SciTech Publishing, 1998.

[28] D. Maiwald and D. Kraus, "Calculation of moments of complex Wishart and complex inverse Wishart distributed matrices," IEE Proceedings Radar, Sonar and Navigation, vol. 147, pp. 162-168, Aug. 2000.

[29] D. R. Cox and N. Reid, "A note on the difference between profile and modified profile likelihood," Biometrika, vol. 79, no. 2, pp. 408-411, 1992.

[30] S. E. Stern, "A second-order adjustment to the profile likelihood in the case of a multidimensional parameter of interest," Journal of the Royal Statistical Society. Series B (Statistical Methodology), vol. 59, no. 3, pp. 653-665, 1997.

[31] T. A. Severini, "Likelihood functions for inference in the presence of a nuisance parameter," Biometrika, vol. 85, no. 3, pp. 507-522, 1998

[32] P. McCullagh and R. Tibshirani, "A simple method for the adjustment of profile likelihoods," Journal of the Royal Statistical Society. Series B (Methodological), vol. 52, no. 2, pp. 325-344, 1990

[33] O. E. Barndorff-Nielsen, "On a formula for the distribution of the maximum likelihood estimator," Biometrika, vol. 70, no. 2, pp. 343365,1983

[34] A. D. C. Nascimento, R. J. Cintra, and A. C. Frery, "Hypothesis testing in speckled data with stochastic distances," IEEE Transactions on Geoscience and Remote Sensing, vol. 48, pp. 373-385, Jan. 2010.
[35] A. C. Frery, J. Jacobo-Berlles, J. Gambini, and M. Mejail, "Polarimetric SAR image segmentation with B-splines and a new statistical model," Multidimensional Systems and Signal Processing, vol. 21, pp. 319-342, 2010.

[36] European Space Agency, Polarimetric SAR Data Processing and Educational Tool (PolSARpro), 4th ed. European Space Agency, 2009. [Online]. Available: http://earth.esa.int/polsarpro/

[37] A. C. Frery, A. H. Correia, and C. C. Freitas, "Classifying multifrequency fully polarimetric imagery with multiple sources of statistical evidence and contextual information," IEEE Transactions on Geoscience and Remote Sensing, vol. 45, pp. 3098-3109, Oct. 2007.

[38] L. Bombrun and J. M. Beaulieu, "Fisher distribution for texture modeling of polarimetric SAR data," IEEE Geoscience and Remote Sensing Letters, vol. 5, pp. 512-516, Jul. 2008.

[39] L. Bombrun, G. Vasile, M. Gay, and F. Totir, "Hierarchical segmentation of polarimetric SAR images using heterogeneous clutter models," IEEE Transactions on Geoscience and Remote Sensing, vol. 49, pp. 726 -737, Feb. 2011.

[40] S. M. Kay, Fundamentals of Statistical Signal Processing, Volume 1: Estimation Theory. Pearson Education, 1998.

[41] D. R. Cox and N. Reid, "Parameter orthogonality and approximate conditional inference," Journal of the Royal Statistical Society. Series B (Methodological), vol. 49, no. 1, pp. 1-39, 1987.

[42] R. A. Johnson and D. W. Wichern, Applied Multivariate Statistical Analysis, ser. Prentice-Hall series in statistics. Prentice-Hall, 1988.

[43] G. A. F. Seber, A Matrix Handbook for Statisticians, 1st ed. New York, NY, USA: Wiley-Interscience, 2007.

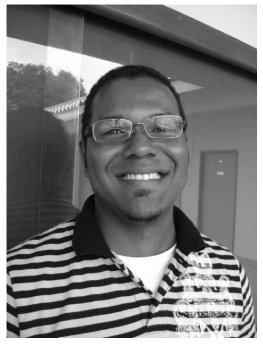

Abraão D. C. Nascimento holds B.Sc. M.Sc. and D.Sc. degrees in Statistics from Universidade Federal de Pernambuco (UFPE), Brazil, in 2005 , 2007, and 2012, respectively. In 2013, he joined the Department of Statistics at UFPB as Adjoint Professor. His research interests are statistical information theory, inference on random matrices, and asymptotic theory.

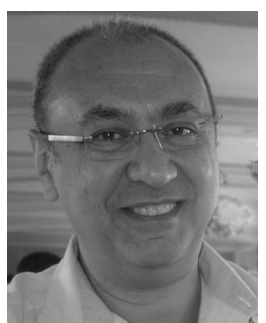

Alejandro C. Frery (S'92-SM'03) received the B.Sc. degree in Electronic and Electrical Engineering from the Universidad de Mendoza, Mendoza, Argentina. His M.Sc. degree was in Applied Mathematics (Statistics) from the Instituto de Matemática Pura e Aplicada (IMPA, Rio de Janeiro) and his Ph.D. degree was in Applied Computing from the Instituto Nacional de Pesquisas Espaciais (INPE, São José dos Campos, Brazil). He is currently the leader of LaCCAN - Laboratório de Computação Científica e Análise Numérica, Universidade Federal de Alagoas, Maceió, Brazil. His research interests are statistical computing and stochastic modelling.

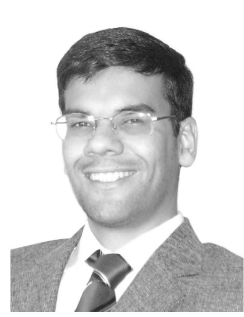

Renato J. Cintra (SM'10) earned his B.Sc., M.Sc., and D.Sc. degrees in Electrical Engineering from Universidade Federal de Pernambuco, Brazil, in 1999, 2001, and 2005, respectively. In 2005, he joined the Department of Statistics at UFPE. During 2008-2009, he worked at the University of Calgary, Canada, as a visiting research fellow. He is also a graduate faculty member of the Department of Electrical and Computer Engineering, University of Akron, $\mathrm{OH}$. His long term topics of research include theory and methods for digital signal processing, communications systems, and applied mathematics. 